\title{
Über Lungensteine.
}

\author{
Vom leitenden Arzte Dr. A. Scherer. \\ (Aus der Kronprinzessin Cäcilic-Heilstätte zu Mühltal, Kreis Bromberg.)
}

(Eingegangen 6. 6. 1921.)

Am 29. Juli $1920 \mathrm{kam}$ ein 45 Jahre alter Ansiedler aus dem Nachbarkreise zu mir in die Sprechstunde, übergab mir cine Karte seines Hausarztes und einen kleinen Fremdkörper, den er kürzlich ausgehustet hatte. Der Inhalt der Karte war, nach Ausscheidung kleinerer Mitteilungen persönlicher Art und der üblichen Höflichkeitsbezeigungen, folgender:

„Herr Ansiedler S. aus B. hat zwei Frauen an Tuberculosis pulmonum verloren. Seit Frühjahr 1920 litt er unter Beginn mit Schüttelfrost an akuter Pneumonie und Fötor, Fieber unbestimmter Art, Nachtschweißen. Am 21. Juli ist plötzlich unter reichlichem Fluor fäkulenter Flüssigkeit, starkem Husten, Erstickungsgefühl beifolgendes Corpưs alienum ausgehustet worden (leider nur der nach starkem Pulen und Spülen restierende Kern wurde mir eingebracht). Danach ist auch Meatus narium frei, der vorher öfters verlegt war, oh ne Schnupfen. Das vorher stets kränkelnde Aussehen (ein Kollega dachte an Malaria) ist seit Ausstoßung des Corpus alienum mit einem Schlage gebessert, auch der Catarrhus pulmonum bessert sich.

Ich schicke Ihnen den Patienten zu zur speziellen Nachuntersuchung. Bitte erklären Sie mir die merkwürdige Anamnese usw.

Dem Patienten hatte der Kollege noch gesagt, er müsse früher einmal ein kleines Knochenstiuck verschluckt haben, das in die Lunge gekommen sei und dort die zunächst rätselhafte Erkrankung hervorgerufen habe.

Die Untersuchung der Lungen des Kranken ergab außer leichten bronchitischen Geräuschen über beiden Unterlappen völlig normale Verhältnisse, insbesondere keinerlei Anhaltspunkte, welche die Annahme des Vorhandenseins einer tuberkulösen Lungenerkrankung gerechtfertigt hätten. Lediglich in der rechten Hilusgegend war hinten eine mäßige Dämpfung nachweisbar, desgleichen mäßig stark abgeschwächtes Atmungsgeräusch, aber keine Bronchophonie, kein verstïrkter Stimmfremitus, auch keine Verstärkung der Flüster. stimme. Eine Röntgenuntersuchung konnte aus äußeren Gründen - wir waren an dem Tage ohne elektrischen Strom - leider nicht stattfinden. Die Untersuchung der Nase ergab leichte Vergrößerung der unteren Muscheln, sonst normale Verhältnisse. Das Corpus alienum war $10 \mathrm{~mm}$ lang, $5 \mathrm{~mm}$ breit und 4,5 $\mathrm{mm}$ dick, von unregelmäßiger Gestalt, aber nirgends mit scharfen Zacken versehen, graubraun; Gewicht $102 \mathrm{mg}$. .Auf Zusatz von reiner Schwefelsäure löste es sich unter lautem Zischen bis auf geringe Sandreste auf, bestand also 
vorwiegend aus kohlensaurem Kalk. Irgendein Strukturgerüst blieb nicht zurück. Die Annahme, daß es sich um ein verschlucktes bzw. aspiriertes Stückchen Knochen oder Knorpel handelt, fällt damit. Der ausgehustete Körper ist vielmehr offenbar ein Lungen-oder Bronchialstein. Höchstwahrscheinlich stellt er den Rest einer verkalkten und nach dem Bronchialsystem durchgebrochenen Bronchialdrüse dar, wurde in die Tiefe des Bronchus aspiriert, rief dann einen pneumonischen Herd und im Gefolge einen kleinen Lungenabszeß hervor. Letzterer heilte nach Ausstoßung des Fremdkörpers sofort ab. Dies ist die einzige Erklärung, die ich für das Krankheitsbild zu geben vermag Da in der Nase nichts weiter zu finden war, als leicht vergrößerte untere Muscheln, nehme ich an, daß das vorangegangene Verlegtsein der Nasengänge nur eine indirekte Folge des Lungenabszesses infolge gesteigerter Kongestion in den gesamten Luftwegen war und daß insbesondere kein Corpus alienum in der Nase selbst bestanden hatte, zumal der Kranke angab, niemals eitrigen oder sonstigen stärkeren Ausfluß aus der Nase beobachtet zu haben. Auch fanden sich keinerlei Anzeichen dafür, daß etwa von einer der Muscheln oder von der Nasenwandung ein Sequester abgestoßen und in die Bronchien aspiriert worden sein könnte.

Dieser Fall gab mir Anlaß, meine 16000 Krankengeschichten der Kronprinzessin Cäcilie-Heilstätte und der von mir ebenfalls geleiteten Wohlfahrtsstelle für Lungenkranke in Bromberg nach beobachteten Lungensteinen zu durchsuchen, um so mehr, als ich gerade in jener Zeit eine Kranke aus Kongreß. polen hier liegen hatte, welche auch immer rätselhaftes Fieber zeigte, das sofort nach dem Auswerfen kleiner Lungensteine für kurze Zeit völlig schwand, um dann, meist ganz plötzlich mit Schüttelfrösten, wiederzukehren, bis ein neuer Stein ausgehustet war. Ich fand, außer einer Reihe von Fällen, in denen die Kranken angaben, Steine oder sogenannten Lungengrie $\$$ ausgehustet zu haben, ohne daß ich selbst diese Konkremente gesehen hätte, im ganzen 21 Fälle (außer dem bereits erwähnten Ansiedler) von Lungensteinen. Aus meiner früheren Tätigkeit an der Heilstätte Ruppertshain i. T. erinnere ich mich, daß dort im Laboratorium eine ganze Sammlung von zum Teil recht großen Lungensteinen aufbewahrt wurden. Ferner habe ich in Beelitz in den Jahren 1902 bis 1904 einige Lungensteine unter Beihilfe eines Kollegen vom Sanatorium, der früher Apotheker und mit solchen Untersuchungen vertraut war, analysiert. Das Ergebnis dieser Analysen ist mir leider verloren gegangen, die Veröffentlichung damals aus äußeren Gründen unterblieben. So viel ich mich erinnere, waren die Hauptbestandteile der dort gewonnenen Steine kohlensaurer und phosphorsaurer Kalk. Hierselbst hat mein leider so früh verstorbener damaliger Assistent Dr. Seybim Frühjahr 1906 drei hier gewonnene Steine analysiert. Das Ergebnis der Analyse wird unten bei den betreffenden Krankengeschichten mitgeteilt werden. Bei den übrigen Steinen beschränkte ich mich auf die Feststellung, daß der Haupt- oder doch ein wesentlicher Bestandteil derselben kohlensaurer Kalk ist, um vor allem die mehrfach von den Kranken geäußerte Ansicht, es handle sich um ,verschluckte" und wieder ausgehustete Knochenstückchen zu widerlegen. Mehrere Steinchen habe ich mit Salpeter. säure entkalkt und auf das Vorhandensein von Tuberkelbazillen untersucht, ohne solche jemals finden zu können (vgl. Nägelsbach, Husten, Auswurf und 
Atemstörungen in Brauer-Schröder-Blumenfeld, Handbuch der Tuberkulose, 2. Bd., 1914). Ein richtiges Strukturgerüst konnte ich bei keinem meiner Steine feststellen. Die Versteinerung ging also offenbar in allen Fällen recht weit zurück, oder es hat sich überhaupt nicht um Versteinerung vorhandenen Gewebes, sondern um Ablagerung steinbildender Substanzen in bereits vorhandenen Hohlräumen gehandelt. Gegen letztere Auffassung würde wieder die unregelmäßige Gestalt und rauhe Oberfläche aller meiner Steine sprechen. Denn Kavernen, seien sie nun in Bronchialdrüsen oder im Lungenparenchym, sind meist glattwandig.

Wenn unter 16000 Kranken nur 21 Fälle mit sicheren Lungensteinen beobachtet werden, also nur $0,13 \%$, so zeigt dies, daß Sahli (Lehrb. d. klin. Untersuchungsmethoden, 4. Aufl.) mit seiner Bemerkung, daß sich ,,in seltenen Fällen" in den Lungen kalkige Konkremente bilden, durchaus Recht hat. Ob die Pathologen bei Sektionen häufiger Lungensteine im Parenchym feststellen, weiß ich nicht, ich konnte darüber in der mir zur Verfügung stehenden Literatur nichts finden. Jemals selbst bei einer Sektion einen Lungenstein gesehen zu haben, kann ich mich nicht entsinnen. Kleinere Konkremente werden auch häufig genug übersehen werden, wenn nicht gerade besonders auf ihr etwaiges Vorhandensein geachtet wird.

Zunächst möchte ich einige der markantesten meiner Fälle hier kurz anführen - alle diese Krankengeschichten ausführlich mitzuteilen, hätte wohl keinen Zweck, da es sich doch mehr oder minder um Wiederholung schon gesagten handeln würde.

In dem oben bereits angezogenen Falle handelt es sich um eine 34 Jahre alte berufslose, unverheiratete Dame, Antonia A. aus Wloclawek, erblich nicht belastet, vor $11 \mathrm{Mo}$. naten mit Husten und Auswurf erkrankt, wiederholt Lungenblutungen, häufig längerdauerndes Fieber, welches nach Aushusten kleiner Steinchen stets für einigo Zeit verschwindet. Aufnahme in die Heilstätte am 27. 5. 1920. Pulmones: Stadium III, beiderseits der ganze Oberlappen erkrankt, rechts deutliche Kavernensymptome. Daneben Ulcus ventriculi (Boassche Druckpunkte deutlich). T.B. + Fieber bis $39,2^{\circ}$ in ore. 5. 6. 1920: Aushusten eines bohnengroßen Lungensteins, $325 \mathrm{mg}$ schwer, sehr porös, von unregelmäßiger Gestalt, nach Auswaschen rein weiß, Größe $10 \mathrm{mal} 9,5 \mathrm{mal} 7 \mathrm{~mm}$. Hauptbestandteil kohlensaurer Kalk. Die Temperatur, die am 5. 6. morgens noch 38,2 in ore betragen hatte, fiel abends auf 37,6. Dann blieb die Kranke fieberfrei. Lungenbefund unverändert.

10. 6. 1920: Nachmittags 4 Uhr Schtittelfrost, Temp. 39,8. Lungenbefund heute und folgende Tage unverändert.

15. 6.: Aushusten eines etwa erbsengroßen Lungensteinchens unter mäßig starker Blutung. Temp. fällt auf 37,3 in ore. Lungenbefund unverändert.

17. 6.: Abends 5 Uhr neuer Schüttelfrost. 39,4 .

21. 6.: Wieder ein erbsengroßer Iungenstein, Abfall der Temperatur auf 37,4, die folgenden Tage hö̈hste Temperatur 37,2 im Munde.

26. B.: Erncuter Schüttelfrost, 40,0.

28. 6.: Leichte Lungenblutung, wobei nach Angabe der Kranken wieder ein kleiner, leider mit dem Auswurf weggegossener Stein abging.

29. 6.: Höchste Temperatur 37,2. Subjektives Wohlbefinden. Lungenbefund unverändert. Die Kranke stand einige Tage später stundenweise auf und machte Licgekur auf dem Zimmer, bis

7. 7. neuer Schüttelfrost eintrat, abends 39,0. Das Fieber blieb dann trotz regelmäBiger Darreichung von dreimal täglich 0,3 Chininum tannicum bis Mitte Juli zwischen 39,0 und 40,0, ohne daß auf den Lungen irgendeine Änderung des Befundes nachzuweisen gewesen wäre und ohne daB wiederholte Röntgendurchleuchtungen Aufklärung gebracht hätten - 
es fanden sich lediglich größere Drüsenpackete in der Hilusgegend, daneben derbe grobfleckige Verdunkelung beider Oberlappen bei vollkommen freier Zwerchfellbewegung. Von Mitte Juli ab ging die Temperatur langsam zurück und hielt sich von da an um 38,0.

24: 7.: Plötzlich Abfall der Temperatur auf 37,3 unter Aushusten eines linsengroßen Steinchens. Die Kranke kam dann bald wieder außer Bett und fühlte sich dabei wohl.

4. 8. 1920: Neuerdings Schüttelfrost, Temp. 39,8. Andauernd hohes Fieber bis

9. 8. abends 40,1. In der Nacht vom 9. zum 10. 8. soll wieder ein etwa linsengroßes Steinchen unter mäBig starker Lungenblutung ausgehustet worden sein, das ich selbst nicht zu sehen bekam.

10. 8.: Höchste Temperatur 37,2 .

13. 8. reiste die Kranke, weil sie von ihrem „Steinleiden“, wie sie es nannte, doch nicht befreit würde, nach Hause. Das Magengeschwür war längst abgeheilt, der Lungenbefund zeigte keine Anderung.

Wie die von der Kranken mitgebrachten Aufzeichungen ihrer dreimal täglich gemessenen Körpertemperatur ergaben, zeigte die Fieberkurve schon in den Monaten vor ihrer Aufnahme einen ähnlichen Verlauf, wie hier selbst. Irgendein Zusammenhang der Schüttelfröste und der Elimination der Steine mit den in diesem Falle ganz regelmäßigen Menses bestand nicht.

Offenbar handelte es sich hier nicht um Parenchymsteine, sondern um durchgebrochene, verkalkte Bronchialdrüsen. Die in die Luftwege eingedrungenen und natürlich als Fremdkörper wirkenden Steinchen riefen kleine, örtlich begrenzte Pneumoniern (Schüttelfröste!) hervor, es kam vermutlich zur Bildung kleiner Lungenabszesse, nach deren Durchbruch in den Bronchus die Pneumonieen abklangen. Anders kann ich mir dieses Krankheitsbild nicht erklären.

Die übrigen Fälle, die ich beobachten konnte, liegen einfacher.

Marie G., 19 Jahre alt, Verkäuferin aus Lissa i. P., aufgenommen am 26. 10. 1908, erblich nicht belastet, seit Januar $1908 \mathrm{krank}$, gibt an, schon vor der Aufnahme in die Heilstätte öfters kleine Steinchen ausgehustet zu haben. Außer schwerer Tuberkulose der Lungen, die rechts den ganzen Ober- und Mittellappen, links den ganzen Oberlappen er. griffen hatte, bestand ein linsengroßes Geschwür an der rechten Stimmlippe, hochgradige Anämie, leichte Neurasthenie und, so lange die Kranke hier weilte, andauernd Fieber, das sich in den Abendstunden zwischen 37,6 und 39,0 hielt. Röntgendurchleuchtung ergab derbe Verdichtungsherde in der ganzen rechten Lunge und im linken Oberlappen, sowie sehr deutliche Drüsenschatten am Hilus.

Am 29. 10. 1908 hustete die Kranke mit ihrem stark eitrigen, massenhaft Tuberkelbazillen enthaltenden Sputum fünf Lungensteine von Linsen- bis Erbsengröße aus. Die Konkremente waren unregelmäßig gestaltet, nach gründlicher Reinigung weiß. Bei Zusatz von Schwefelsäure zischten sie auf, der Rückstand war braungrau, wie zerfallender Waschschwamm, in dessen Poren sandige Teile eingelagert waren. Beim Waschen zerfiel dieses Gerüst vollständig in eine pulverige Masse. Zwei Steinchen wurden mit Salpetersäure zu mazerieren versucht, irgendeine Struktur ließ sich aber nicht nachweisen. Am 15. 11. hustete die Kranke nochmals einen erbsengroßen Stein aus unter gleichzeitiger Rötung des Sputums. Die Blutspuren versehwanden schon am nächsten Tage aus dem Auswurf. Am 30. 11. mußte die Kranke entlassen werden, da keine Aussicht bestand, einen Erfolg zu erzielen.

Vermutlich handelte es sich auch in diesem Falle um durchgebrochene verkalkte Bronchialdrüsen. Da aber an einem Tage gleich fünf Steine auf einmal ausgeworfen wurden, muß man wohl auch an die Möglichkeit denken, daß hier Parenchymsteine vorlagen. Ein Abfallen der Körpertemperatur erfolgte nach Ausstoßung der Steinchen nicht.

Ein weiterer Fall ist insoferne interessant, als es sich um eine Kranke mit nur sehr geringfügigen Veränderungen über den Lungen handelte. 
Minna K., 23 Jahre alte Köchin aus Kruglanken, aufgenommen am 11. 12. 1905, erblich nicht belastet. Lungenbefund: Geringe Schallverkürzung und rauhes Atmen mit vereinzeltem Knacken über der linken Lungenspitze, Außer geringer Anämie keine Komplikationen. Dauernd fieberfrei. Tuberkulinprobe positiv, 38,4 in ore nach 0,5 Alttuberkulin.

5. 3. 1906: Aushusten mehrerer kleiner Lungensteine (hirsekorn- bis linsengroß) ohne alle Beschwerden, auch kein Auswurf.

14. 3. 1906: Aushusten eines reichlich bohnengroßen Steins von unregelmäßiger Gestalt, weißer Farbe. Gewicht des Steines $520 \mathrm{mg}$.

Die von Herrn Dr. Seyb vorgenommene quantitative Analyse ergab:

$$
\begin{aligned}
& \text { Kohlensaurer Kalk . . . . . . . . . . . . } 204 \text { mg, } \\
& \text { Phosphorsaurer Kalk . . . . . . . . . . } 112 \text {, } \\
& \text { Kieselsäure . . . . . . . . . . . . . . . } 17 \text { " } \\
& \text { Kohlensaure Magnesia . . . . . . . . . } 23 \text { " } \\
& \text { Fette und Cholestearine . . . . . . . . . } 88 \text { ", } \\
& \text { Verlust . . . . . . . . . . } 76 \text { ", }
\end{aligned}
$$

Die Analyse ergab also im wesentlichen dieselben Bestandteile, wie die in der Literatur bekannt gegebenen Untersuchungsbefunde anderer Autoren, auf die ich unten noch zurückkommen werde. Nur herrschte in diesem Falle der kohlensaure Kalk gegenüber dem phosphorsauren vor. Von Interesse ist, da $\beta$ in dem Falle die Steine ausgehustet wurden, obwohl nicht nur keinerlei Zerfallserscheinungen auf den Lungen nachweisbar waren, sondern auch gar keine subjektiven Beschwerden bestanden. Nach Form und Zusammensetzung der Steine konnte aber kein Zweifel bestehen, daß es sich um aus den tieferen Luftwegen stammende Konkremente handelte, und zwar möchte ich auch in diesem Falle wieder annehmen, daß es Reste von verkalkten Bronchialdrüsen waren, obwohl es kaum erklärlich ist, wie solche zum Teil doch ziemlich großen Steine durch die Wandung der Bronchien bzw. der Trachea wandern können, ohne wenigstens eine leichte Blutung oder doch Auswurf hervorzurufen. $\mathrm{Ob}$ bei der Kranken besonders große Bronchialdrüsen bestanden, konnte ich leider nicht feststellen. Mittels der üblichen klinischen Untersuchungsmethoden waren solche nicht nachweisbar, und über eine Röntgeneinrichtung verfügte ich damals noch nicht.

Einen besonders großen Stein, den größten, den ich mich jemals gesehen zu haben entsinne, verdanke ich einem Kranken der Wohlfahrtsstelle für Lungenluranke zu Bromberg.

Boleslaus Cz., Sattlergeselle aus Bromberg, 27 Jahre alt, beide Eltern an 'Tuberkulose gestorben, Ehefrau und drei Kinder lungenkrank, hat seit drei Jahren häufig Lungenblutungen. Auswurf enthält Tuberkelbazillen. Der Kranke fiebert fast ständig und ist dauernd und fast völlig arbeitsunfähig.

Am 2. 1. 1906 brachte mir seine Frau einen großen Stein und einen in mindestens einem Viertelliter reinen Blutes schwimmenden Bronchialbaum, dic ihr Mann am Tage vorher nach einem schweren Erstickungsanfall, welcher nach einer profusen Lungenblutung aufgetreten war, ausgehustet hatte. Ob erst der Stein oder zunächst der Bronchialbaum aus. geworfen worden war, vermochte ich nicht festzustellen. Sofort sei dem Kranken leichter geworden.

Der Kranke ging im Juni 1907 an sciner schweren Lungentuberkulose zugrunde. Von weiteren ausgehusteten Steinen oder Bronchialbäumen wurde mir nichts mehr mitgeteilt Ich selbst hatte den Kranken bis kurz vor seinem Tode regelmäßig alle ein bis zwei Monate in der Sprechstunde der. Wohlfahrtsstelle untersucht. 
Der Stein war, wie fast alle von mir beobachteten Lungenkonkremente, unregelmäßig geformt, $15 \mathrm{~mm}$ lang, $12 \mathrm{~mm}$ breit, $8,5 \mathrm{~mm}$ dick. Der Bronchialbaum war reich verzweigt und sehr schön erhalten, in seiner größten Länge maß er $135 \mathrm{~mm}$, im Wasser flach ausgebreitet war er $98 \mathrm{~mm}$ breit, also ein recht stattliches Präparat dieser Art, das mir leider, nachdem ich es in Chromsäure gehärtet hatte, bei mehrfachen Transporten zu Demonstrationszwecken derart zerbrach, daß es schließlich jeden Wert verlor. Am obersten Ende war das Gerinnsel $9 \mathrm{~mm}$ stark, dürfte sich also bis unmittelbar an die Bifurkation erstreckt haben. Ich vermute deshalb, daß der Stein, der $976 \mathrm{mg}$, also beinahe $1 \mathrm{~g}$ wog, ebenfalls aus einer verkalkten und nach der Trachea durchbrochenen Hilusdrüse stammte.

Nach der von Herrn Kollegen Seyb vorgenommenen Analyse enthiclt der Stein:

$$
\begin{aligned}
& \text { Phosphorsauren Kalk . . . . . . . . } 470 \text { mg, } \\
& \text { Phosphorsaure Magnesia . . . . . . . } 11 \text { ", } \\
& \text { Kohlensauren Kalk . . . . . . . . . . 220 ,, } \\
& \text { Kieselsäure . . . . . . . . . . . . . . . Spuren, } \\
& \text { Fett und Cholestearine . . . . . . . . . } 85 \mathrm{mg} \text {, } \\
& \text { Verlust bzw. unbestimmbar . . . . . . . . } 190 \text {,, } \\
& \text { Gewicht des Steines: } 976 \mathrm{mg} \text {. }
\end{aligned}
$$

Also auch hier vorherrschend Kalksalze, daneben verhältnismäßig viel (fast 10\%!) Fett und Cholestearine. Der große ,Verlust" bei der Analyse wird den vorwiegend chemisch arbeitenden Lesern auffallen, erklärt sich aber daraus, daß der Untersucher derartige Arbeiten nur aus Liebhaberei betrieb und es ihm deshalb wohl an der erforderlichen Gewandtheit und Übung gebrach, um die ihm hier zur Verfügung stehenden und für solche Zwecke völlig ausreichenden Einrichtungen mit noch größerem Glück zu benützen. Ich bin mit dem erreichten völlig zufrieden, da ja der Zweck, über die ungefähre Zusammensetzung der Steine unterrichtet zu sein und vor allem den Beweis dafür zu haben, daß es sich tatsächlich um Lungensteine und nicht etwa um von außen aspirierte Fremdkörper handelt, vollständig erreicht ist.

Noch zweier Fälle will ich erwähnen, weil sie in gewissem Sinne interessant sind.

Verkäuferin Ottilie W., 26 Jahre alt, aus Charlottenburg, aufgenommen am 25. 5. 1912, entlassen am 22. 2. 1913. Schwere, vorwiegend einseitige Erkrankung, die ganze rechte Lunge mit Kavernen durchsetzt. Nach Anlegung eines rechtsseitigen Pneumothorax besserte sich der vorher recht trostlose Zustand der Kranken. Sechs Wochen nach der Anlegung des Pneumothorax wurde ein $320 \mathrm{mg}$ schwerer Lungenstein ausgehustet, der verhältnismäßig wenig scharfe Kanten und Ecken zeigte, aber auch nicht abgerundet war, sondern mehr Würfelform besaß. Er war sehr porös und enthielt vorwiegend phosphorsauren Kalk. Besondere Beschwerden waren mit dem Aushusten nicht verbunden, auch keine Blutung, keine Steigerung des damals — unter Pneumothoraxwirkung — schon recht spärlich gewordenen Auswurfs. Nach der Elimination des Steins fühlte sich die Kranke weder besser noch schlechter als vorher. Einige Monate nach der Entlassung aus der Heilstätte ging sie an Darmtuberkulose zugrunde.

Der Stein war nicht ganz so porös, wie die andern von mir beobachteten, er war zwar nicht glattwandig, aber in der Oberfläche nicht so zerrissen und zerklüftet, wie die übrigen. Es wäre also nicht ganz ausgeschlossen, daß er 
aus einer Kaverne stammte. Die Mazeration der Oberfläche müßte dann durch etwaigen Säuregehalt der Kavernenflüssigkeit hervorgerufen worden sein. Vielleicht handelt es sich aber auch hier um die Reste einer verkalkten Bronchial. drüse. Die Frage, ob der Pneumothorax auf die Ausscheidung des Steines irgendwelchen Einfluß hatte, wage ich nicht zu entscheiden, möchte sie aber nicht ohne weiteres verneinen. Es ist immerhin möglich, daß bei dem damals von mir auch schon im Beginn der Pneumothoraxbehandlung regelmäßig angewandten sehr hohen Uberdruck der Stein aus dem Lungengewebe direkt herausgepreßt wurde. Ebensogut wäre es aber in solchen Fällen möglich, daß ein nahe der Pleura gelegener Stein in den Pleuraraum, also in den Pneumothorax hinein ausgepreßt wird und dort durch mitgerissene Eitererreger ein Empyem hervorruft, also zum Pyopneumothorax führt. Sollte nicht mancher trotz strengster Asepsis bei den Nachfüllungen aufgetretene Pyopneumothorax auf diese Weise erklärt werden können? Jedenfalls haben mich diese Erwägungen veranlaßt, späterhin bei der Pneumothoraxbehandlung erst dann und auch nur ausnahmsweise zur Anwendung hohen Drucks zu schreiten, wenn ich glaubte, damit rechnen zu dürfen, daß durch monatelange Einwirkung des Pneumothorax auf die Lungenpleura eine erhebliche Verdickung und Verhärtung derselben eingetreten sein mußte und daß die erfahrungsgemäß bei der Lungenkollapstherapie eintretende bindegewebige Entartung des Lungenparenchyms in den kollabierten Lungenteilen bereits einen hohen Grad erreicht hatte, so daß das Durchwandern von etwa vorhandenen Steinen nicht mehr so leicht möglich sein könnte. Gelegenheit, einen von mir behandelten Pneumothoraxfall zu sezieren, hatte ich ja glücklicherweise bis jetzt noch nicht, ich kann also nicht sagen, ob etwa öfters kleine Steine nach dem Pneumothorax durchbrechen, ohne schwerere Erscheinungen zu machen. In der Literatur konnte ich in den Sektionsberichten nichts davon finden, daß bei einem Pneumothorax jemals Steine festgestellt worden wären.

Der letzte Fall, den ich noch anführen möchte, erscheint mir deshalb bemerkenswert, weil sich die betreffende Kranke noch in sehr jugendlichem Alter befand.

Frieda W., Schülerin aus Gnesen, 121/2 Jahre alt, aufgenommen am 16. 10. 1913, zeigte bei der Aufnahme in die Heilstätte keine deutlichen Erscheinungen über den Lungen, auch im Röntgenbilde nicht. Dagegen waren ausgedehnte Bronchialdrüsentumoren klinisch und mittels Röntgenstrahlen deutlich nachweisbar. Die Kranke neigte stark zu Fieber, war außergewöhnlich frühzcitig (mit ll Jahren) völlig entwickelt und bot trotz des fehlenden Lungenbefundes prognostisch keine günstigen Aussichten.

Schon am 3. 6. 1914 mußte sie zum zweiten Male aufgenommen werden, weil sich ihr Leiden in der Zwischenzeit bedeutend verschlimmert hatte. Fs war Husten und Auswurf, zeitweilig mit Blut dazwischen aufgetreten, und wenige Tage nach ihrem 13. Geburtstage hatte sie einen großen, unregelmäßig gestalteten Stein ausgehustet, nach dessen Ausstoßung das vorher bestandene Fieber rasch verschwunden sein soll (Zufall oder Wirkung?). Bald nach der Elimination des Konkrementes, das sie mitbrachte, kam sie wieder in die Heilstätte, wo wir sie - es bestand jetzt eine ausgesprochene Erkrankung beider Oberlappen mit positiven Bazillenbefund, aber kein Fieber - mit Perlsuchtalttnberkulin behandelten und in viermonatigem Aufenthalt eine wesentliche Besserung erzielten. Die Tuberkelbazillen verschwanden aus dem Auswurf.

Der Stein wog $440 \mathrm{mg}$, war $12 \mathrm{~mm}$ lang, $10 \mathrm{~mm}$ breit und 7,5 mm dick, von fast reinweißer Farbe. Er enthielt vorwiegend phosphorsauren, daneben 
kohlensauren Kalk und, nach Extraktion mit Äther, verhältnismäßig viel Fett. Eine quantitative Analyse konnte nicht stattfinden. Nach Entkalkung verblieb ein graubräunlicher Detritus, in welchem Tuberkelbazillen nicht festzustellen waren. Auch konnte keinerlei Gewebsstruktur aufgefunden werden. - Im Röntgenbilde waren Schatten, welche von Steinen hervorgerufen sein konnten, nicht nachzuweisen.

Auch hier bin ich der Ansicht, daß es sich um eine verkalkte Bronchialdrüse handelte, die nach dem Bronchus durchgebrochen war, vielleicht nachdem sie längere Zeit im Lungengewebe gesteckt und dort einen Entzündungsherd (Pneumonia dissecans nach Rindfleisch?) hervorgerufen hatte. Ob das Durchbrechen des Steins erst zum Einwandern der Tuberkulose in das Lungengewebe und zu ihrem überraschend schnellen Ausbreiten daselbst geführt oder doch wesentlich hierzu beigetragen hat, kann man natürlich nicht entscheiden. Ioh möchte dies aber nicht nur für möglich, sondern nach dem ganzen Krankheitsbilde für wahrscheinlich halten.

Die übrigen Fälle von Lungensteinen, die ich boobachten konnte, bieten keine Besonderheiten, ich kann also auf ihre Wiedergabe verzichten. Nur noch die quantitative Analyse eines Steines von $460 \mathrm{mg}$ Gewicht, die ich ebenfalls Herrn Dr. Seyb verdanke, möchte ich anführen. Der Stein enthielt:

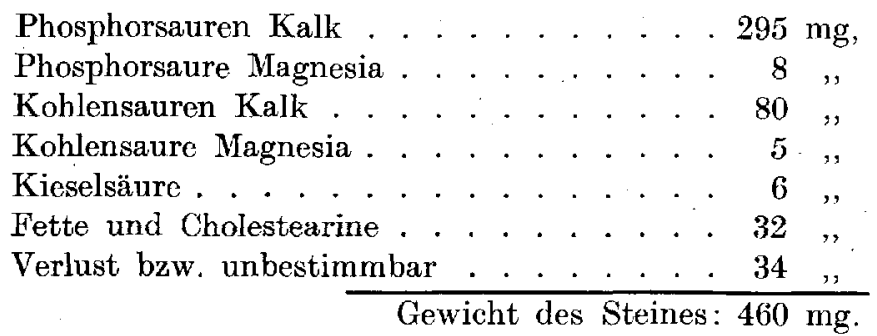

Also auch hier Kalksalze als die hauptsächlichsten Bestandteile.

In der Literatur sind die Lungenkonkremente etwas stiefmüttcrlich behandelt. Bandelier und Röpke, Die Klinik der Tuberkulose, tun ihrer in der ersten und zweiten Auflage - die späteren Auflagen sind mir nicht zu. gänglich - überhaupt keine Erwähnung, in der ersten Auflage von Cornets großem Werk „Die Tuberkulose“ sind sie mit nicht ganz zwei Zeilen abgetan, in der zweiten Auflage kann ich gar nichts darüber finden. Nägelsbach („Husten, Auswurf und Atemstörungen" in Brauer-Schröder-Blumenfelds Handb. d. Tub. 2. Bd., 1914) spricht nur kurz darüber, auch in dem großen Handbuch der inneren Medizin von Mohr und Staehelin sind Bronchialund Lungensteine mit wenigen Zeilen abgehandelt.

Ausführlicher befassen sich damit Ott (Die chem. Pathol. der Tub.), A. Fränkel (Spez. Pathol. u. Ther. der Lungenkrankheiten) und F. A. Hoffmann. (Die Krankheiten der Bronchien). Bei Ott finden wir u. a. zwei Analysen von Lungensteinen zitiert, die ich, zum Vergleich mit meinen oben angegebenen, hier anführen möchte:

Die erste betrifft einen Stein von $3,0 \mathrm{~g}$ Gewicht, der also nach meinen Erfahrungen als außergewöhnlich groß angesehen werden muß und der aus dem 
Lungenparenchym gelöst worden war (Sgarzi, Annal. univ. di Med. Vol. 87, 1837). Die Analyse ergab:

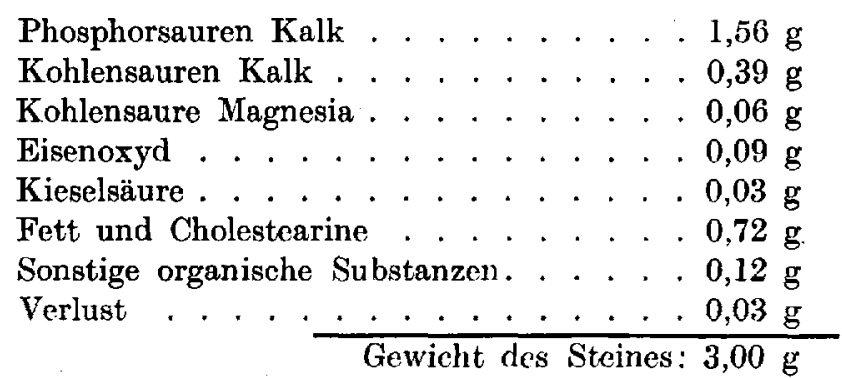

Die Analyse eines aus den Bronchien stammenden Steines, veröffentlicht von Ball et Vee, Soc. de Biol. 1858, von Ott ziticrt nach Poulalion, zeigte folgende Zusammensetzung:

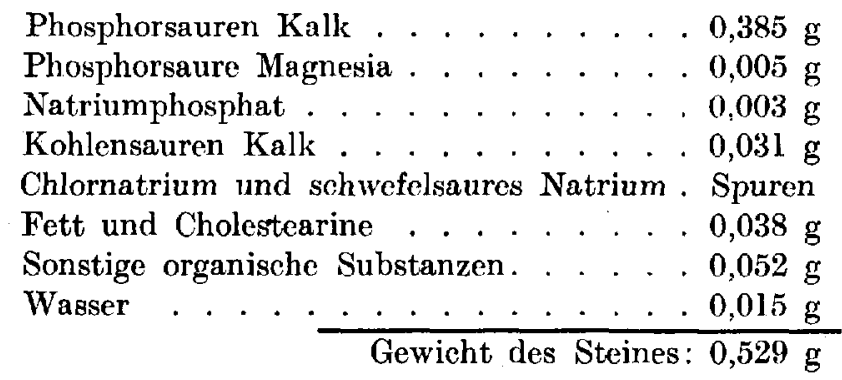

Im wesentlichen ergaben somit diese letzten beiden Analysen einen sehr ähnlichen Befund, wie ihn Scyb bei meinen Steinen erhoben hat: Phosphorsauren bzw. kohlensauren Kalk als Hauptbestandteil, daneben außer einigen ànderen alkalischen Erden etwas Fett und Cholestearine. Auffallend gering war, soweit er nicht vollständig fehlte, bei obigen Analysen der Gehalt an Kieselsäure bzw. kieselsauren Salzen, während Zickgraf (Über die therap. Verw. des kieselsauren Natriums und die Beteiligung der Kieselsäure an der Bildung von Steinen, diese Beitr., Bd. 5) gerade den außerordentlich großen Kieselsäuregchalt $(4,71-12,45 \%$ !) der von ihm untersuchten Steine hervorhebt. An der Verschiedenheit der Ernährung der betreffenden Patienten kann dies meines Erachtens nicht liegen, so viel Kieselsäure, als zur Bildung von Steinen erforderlich ist, findet sich in der mensehlichen Nahrung immer, gleichviel, ob der Kranke Vegetarier ist oder ausgiebigstem Fleischgenuß huldigt. Es müssen also für diese Verschiedenheit der Zusammensetzung der Steine andere, vorerst nicht aufzuklärende Gründe vorliegen.

Über die Art und Weise, wie die Steine entstehen, gehen die Ansichten auseinander. Meine eigenen Beobachtungen und Untersuchungen geben darüber keinen Aufschluß und ich glaube, wir werden bei ausgehusteten Steinen immer mehr oder weniger auf bloße Vermutungen über ihren Ursprung angewiesen sein. Nach meinem Dafürhalten wird es sich meist um die durchgebrochenen Reste verkalkter Hilusdrüsen handeln. Es wird aber nach pathologischen Befunden durchaus nicht selten sein, daß Parenchymsteine oder solche, die: in 
Kavernen entstanden sind, in die Luftwege durchbrechen. Ja, ich möchte es für durchaus möglich erklären, daß sich auch im Lumen der Bronchien selbst ebenso wie in den Nasengängen oder Ausführungskanälen der Speicheldrüsen Steine bilden, wenn diese auch nur klein bleiben dürften (,Lungengrieß"), weil sie ja immer leicht und rasch eliminiert werden können. Nach meinem Dafürhalten dürften aber die Wandungen solcher echten „Bronchialsteine“ nicht derart zerrissen und rauh sein, wie die sämtlichen von mir beobachteten Lungensteine. Rhinolithen und Speichelsteine, soweit ich solche zu sehen Gelegenheit hatte, waren vielmehr immer glatt, abgerundet, ohne scharfe Ecken und Vorsprünge.

Nachweisen lassen sich am Lebenden Lungensteine in situ offenbar nur schwer. Ich selbst kann mich nicht erinnern; bei den vielen tausenden von mir vorgenommenen Röntgenuntersuchungen jemals einen Lungenstein zweifelsfrei im Röntgenbilde nachgewiesen zu haben. Bickel und Grum mach (Berl. klin. Wochenschr., 1908, Nr. 1) beschreiben einen Fall von ,Steinhusten“" in welchem an drei Dutzend stecknadelkopf- bis erbsengroße Steinchen ausgeworfen wurden und bei dem im Röntgenbild Steine deutlich nachzuweisen waren.

Über die Art der Entstehung der Lungensteine gibt A. Fraenkel (l. c.) eine übersichtliche Zusammenstellung. Er unterscheidet:

1. Einfache Kalkkonkremente, die hauptsächlich bei Tuberkulose der Lungen vorkommen, und zwar in den verkästen Herden, in den Bronchialdrüsen, außerdem in bronchiektatischen Kavernen, wenn ihr Inhalt sich nicht entleeren kann, sondern zur Eindickung verurteilt ist.

2. Richtige Petrifikation der Bronchien, wobei ebenfalls verkalkte Reste abgestoßen und ausgeworfen werden können.

3. Diffuse Inkustrationen des Lungengewebes selbst; die or als selten bezeichnet.

4. Pneumonokoniosen, Chalikosis, Anthrakosis. Dabei wird es wohl nur zur Abstoßung kleinster Steinchen kommen können.

5. Echte Knochenbildung an den Bronchialknorpeln, aber auch im Bereiche der Schleimhäute vorkommend, ferner in der Wand von Abszessen.

6. Uratablagerungen bei Gichtikern, die nach Bernstein ebenfalls als Lungensteine ausgeworfen werden können.

7. Zystinkonkremente, wie sie Lubarsch in seiner bronchiektatischen Kaverne beobachtete.

Úber die Symptomatologie der Lungenkonkremente ist nicht viel zu sagen. Charakteristisch ist lediglich das Aushusten der Steinchen oder von Grieß. Die dabei oft auftretenden Begleiterscheinungen (hohes Fieber, allenfalls Schüttelfröste, Husten, Hämoptöen, Dyspnöe, Nachtschweiße) sind in keiner Weise spezifisch aufzufassen.

Eine eigentliche Thera pi e der Lungensteine gibt es nicht. Dieselbe könnte nur symptomatisch sein, sich also z. B. auf die Entfernung eines in der Luftröhre oder einem größeren Bronchus steckenden und nicht spontan expektorablen Steines mittels des Bronchoskops erstrecken. Auch die Linderung quälender Begleitsymptome aller Art fiele hierher. Eine kausale Therapie oder Prophylaxe in der Richtung, daß wir die Bildung von Lungensteinen verhindern oder 
die Auflösung bereits gebildeter befördern wollten, etwa durch saure oder kalkund kieselsäurearme Diät, durch Genuß steinlösender Wasser oder dergleichen, würde meines Erachtens nicht das richtige treffen, da wir doch die Steinbildung, bei Tuberkulose wenigstens, als eine Art Heilungsvorgang auffassen müssen. Es käme für solche therapeutischen Bestrebungen wohl nur die gichtische Form, allenfalls auch noch die in ihrer Ätiologie vorerst ungeklärte, nicht auf Tuberkulose beruhende Phthise calcaire (Bayle, Recherches sur la Phthise pulmonaire, Paris 1810) oder Pseudophthisis calculosa, wie F. A. Hoff mann (1. c.) sie nennt, in Frage.

Aufgabe weiterer Untersuchungen wird es sein, allmählich dahin zu gelangen, daß wir über die Struktur der Lungenkonkremente noch genauer unterrichtet werden. Nur dann haben wir auch die Möglichkeit zur sicheren Feststellung der Herkunft der Steine. Ich habe, wie oben schon erwähnt, eine Reihe von Steinen entkalkt, ohne daß es mir gelungen wäre, Tuberkelbazillen oder eine bestimmte Struktur aufzufinden. Zur Entkalkung wandte ich verschiedene Mittel an: Reine Salpetersäure, Salpetersäure-Alkohol mit Zusatz von Chlornatrium, Ebners Entkalkungsflüssigkeit, 2\% Chromsäurelösung, Holzessig, alles mit dem gleichen Mißcrfolg. Vielleicht beschert uns aber die pathologischchemische Technik bald Methoden, die in dieser Richtung besseres leisten, als die jetzt bekannten. 